Luís Felipe Lopes Lorenzon?

(ㄱ) https://orcid.org/0000-0002-4533-005X

Patrícia Beatriz Pedroso Minossi

Ohttps://orcid.org/0000-0003-0614-661X

Giovana Eliza Pegolo

Ohttps://orcid.org/0000-0003-2636-254

\section{Ortorexia nervosa e imagem corporal em adolescentes e adultos}

\author{
Orthorexia nervosa and body image in adolescents and adults
}

DOI: $10.1590 / 0047-2085000000266$

\section{RESUMO}

Objetivo: Identificar a frequência do comportamento de risco para ortorexia nervosa em uma amostra de indivíduos com idades entre 18 e 60 anos e associar com o estado nutricional (classificação do peso corporal) variáveis sociodemográficas e imagem corporal. Métodos: Trata-se de um estudo transversal constituído por indivíduos de ambos os sexos. Utilizou-se o questionário ORTO-15 (com pontos de corte < 40 e < 35) para a identificação de comportamentos de risco para ortorexia e a Escala de Silhuetas para a imagem corporal. O estado nutricional foi avaliado por meio do índice de massa corporal, com peso e altura autorreferidos. Para a análise dos dados, aplicou-se o teste qui-quadrado, com nível de significância de 5\%. Resultados: Participaram 430 indivíduos, sendo 56,7\% ( $\mathrm{n}=244$ ) mulheres, com idade para ambos os sexos entre 18,1 e 59,9 anos. Constatou-se maior número de participantes com risco para ortorexia ao utilizar o ponto de corte $<40(91,4 \%, n=393)$ quando comparado ao ponto de corte $<35(54,4 \%, \mathrm{n}=234)(\mathrm{p}<0,0001)$. O estado nutricional não esteve associado ao comportamento ortoréxico $(<35, p=0,68 ;<40, p=0,69)$, bem como à imagem corporal, em ambos os sexos $(<40$ e $<35)$. A idade entre 40 e 60 anos associou-se com a presença de ortorexia $(<35)$ ( $p=0,0005)$, enquanto não houve associação com as variáveis sexo, escolaridade, estado civil e renda (< 40 e <35). Conclusão: Os resultados do presente estudo indicam alta frequência de comportamento de risco para ortorexia. Sugerimos a investigação da frequência nos diversos segmentos populacionais e dos fatores associados ao desenvolvimento de atitudes ortoréxicas.

\section{PALAVRAS-CHAVE}

Autoimagem, comportamento alimentar, peso corporal.

\section{ABSTRACT}

Objective: To identify the frequency of risky behavior for orthorexia nervosa in a sample of individuals aged between 18 and 60 years and associate it with nutritional status (classification of body weight), sociodemographic variables and body image. Methods: This is a cross-sectional study of individuals of both sexes. The ORTO-15 questionnaire (with cutoff points $<40$ and $<35$ ) was used to identify risk behaviors for orthorexia and the Silhouette Scale for body image perception. Nutritional status was assessed by body mass index, with self-reported weight and height. For data analysis, the chi-square test was applied, with a significance level of 5\%. Results: 430 individuals participated, being 56.7\% ( $n=244$ ) women, aged between 18.1 and 59.9 years for both sexes. There was a higher number of participants at risk for orthorexia when using the cutoff $<40(91.4 \%, n=393)$ when compared to the cutoff $<35(54.4 \%, n=234)(p<0.0001)$. Nutritional status was not associated with orthorexic behavior $(<35, p=0.68 ;<40, p=0.69)$, as well as body image in both sexes $(<40$ and $<35)$. Age between 40 and 60 years was associated with the presence of Orthorexia $(<35)(p=0.0005)$, while there was no association with gender, education, marital status and income $(<40$ and $<35)$. Conclusion: The results of the present study indicate a high frequency of risk behavior for Orthorexia. We suggest investigating the frequency in the various population segments and the factors associated with the development of orthorexic attitudes.

\section{KEYWORDS}

Self concept, eating behavior, body weight. 


\section{INTRODUÇÃO}

Ortorexia nervosa é o termo que designa indivíduos excessivamente preocupados com o consumo de alimentos saudáveis'. Esse quadro inicia-se de maneira sutil, a partir do desejo de corrigir hábitos alimentares entendidos como ruins ou de melhorar a saúde como um todo², mas acaba por conduzir a pessoa a desenvolver características comportamentais associadas à obsessão pela pureza da alimentação, lutando repetidamente contra o consumo de alimentos com substâncias consideradas impuras ${ }^{3}$.

Essas práticas requerem considerável força de vontade e disciplina, que são reforçadas pelo senso de superioridade em relação àqueles que consomem alimentos tidos como não saudáveis ${ }^{4,5}$. O indivíduo também tem a crença persistente de que tais práticas alimentares são promotoras de saúde, apesar das significativas complicações médicas e evidências de má nutrição ${ }^{6}$, mesmo assim manifestam tendência de encorajar familiares e amigos a seguirem suas "dietas supostamente saudáveis"7.

Nesse contexto, sugere-se que a ortorexia apresente como característica a prática de uma alimentação perfeita e com rigorosas regras dietéticas. Esse perfeccionismo ultrapassa a esfera comportamental e adentra no âmbito emocional, alterando o modo que o indivíduo enxerga o tamanho, a forma, a estrutura de seu corpo, além dos sentimentos em relação a essas características, podendo estar associado com a aparência e o medo do excesso de peso ${ }^{8,9}$.

Estudo realizado com adultos italianos, em 2011, provenientes da população em geral, detectou 57,6\% de indivíduos em risco para ortorexia ${ }^{10}$. Em 2019, entre adultos libaneses, 75,2\% apresentaram comportamentos indicativos de ortorexia"1" No Brasil, para segmentos específicos, estudos realizados com estudantes do curso de Nutrição, reconhecidos como grupo de risco, constataram percentuais de $88,7 \%{ }^{12}$ e $87,2 \%{ }^{13}$ de universitários com comportamento alimentar indicativo do referido quadro.

Cabe registrar que até o momento a ortorexia não está incluída no Manual Diagnóstico e Estatístico de Transtornos Mentais $^{14}$. A fim de compreender e melhor categorizar a ortorexia destaca-se a sobreposição de sintomas incluídos nos quadros de transtorno obsessivo-compulsivo, na anorexia nervosa e na bulimia nervosa, sendo os principais sintomas comuns a ansiedade, a restrição de determinados alimentos, o perfeccionismo e a adoção de comportamentos ritualísticos. Por outro lado, são elencados sintomas exclusivos da ortorexia, especialmente a obsessão por ser saudável ou mais saudável e o sentimento de superioridade e aumento do senso de retidão, sintoma não associado a outros distúrbios alimentares ${ }^{15}$. Destaca-se a ausência de um critério diagnóstico formal para a ortorexia nervosa como fator limitante, e alguns autores indicam como critérios diagnósticos primários: a preocupação obsessiva ou patológica com a alimentação saudável; a presença de consequências emocionais, como angústia e ansiedade, originadas pela não adesão às regras alimentares autoimpostas; prejuízos psicossociais em relação à vida cotidiana, desnutrição e perda de peso ${ }^{16}$.

Este estudo objetivou identificar a frequência do comportamento de risco para ortorexia nervosa em uma amostra de indivíduos com idades entre 18 e 60 anos e associar com o estado nutricional (classificação do peso corporal), imagem corporal e variáveis sociodemográficas.

\section{MÉTODOS}

\section{Amostra}

Trata-se de um estudo transversal, com abordagem quantitativa e amostra por conveniência, constituída por indivíduos, de ambos os sexos, que frequentaram um parque urbano de Campo Grande (Mato Grosso do Sul) durante o período de coleta de dados, realizada em 2018.

Os critérios de inclusão foram: idade igual ou superior a 18 anos e inferior a 60 anos completos. Foram excluídos indivíduos com deficiência física ou gestação, por representarem situações que podem interferir na construção da imagem corporal, e presença de dificuldade intelectual que pudesse comprometer a compreensão do questionário.

Este estudo atende aos princípios éticos para pesquisas envolvendo seres humanos, conforme a Resolução no 466/2012, do Conselho Nacional de Saúde17, e foi aprovado pelo Comitê de Ética em Pesquisa em Seres Humanos da Universidade Federal de Mato Grosso do Sul (Parecer no 2.355.567). Todos os participantes integraram a pesquisa voluntariamente e registraram sua concordância ao assinar o Termo de Consentimento Livre e Esclarecido, apresentado na forma impressa.

\section{Procedimentos}

Para a obtenção dos dados, foram utilizados três instrumentos: o ORTO-15'18, a Escala de Silhuetas, adaptada e validada para a população brasileira ${ }^{19} \mathrm{e}$ um questionário com informações sociodemográficas e nutricionais contemplando data de nascimento, sexo, escolaridade, estado civil, renda familiar mensal e medidas antropométricas autorreferidas. O estado nutricional foi avaliado por meio da classificação do índice de massa corporal (IMC), de acordo com os pontos de corte propostos pela Organização Mundial da Saúde e adotados, no Brasil, pelo Sistema de Vigilância Alimentar e Nutricional, sendo que para os adolescentes foi utilizado o IMC/idade ${ }^{20}$. O cálculo do IMC foi realizado a partir do peso e altura autorreferidos.

\section{Instrumentos \\ ORTO-15}

Para identificar comportamentos indicativos de risco de ortorexia, adotou-se o questionário ORTO-1 $5^{21} \mathrm{com}$ tradução 
e adaptação cultural à realidade brasileira, a fim de aplicá-lo como instrumento de análise do comportamento ortoréxico em estudos populacionais ${ }^{18}$. Esse instrumento é constituído por 15 perguntas, pontuadas por uma escala Likert com quatro opções de respostas com escores de 1 a 4 (Sempre, Frequentemente, Às vezes e Nunca). A pontuação total do questionário, com variação de 15 a 60, é obtida somando a pontuação de cada item²2.

Segundo Donini et al. ${ }^{21}$, a partir da pontuação das respostas do referido questionário, originalmente se indica o ponto de corte $<40$ como sensível para identificar indivíduos em risco. Contudo, estudos mais recentes demonstraram preocupações com a pontuação $<40$ por resultar em falso-positivos e altas porcentagens de indivíduos com comportamento de risco para ortorexia ${ }^{7,10}$. Portanto, sugere-se que a pontuação < 35 apresente maior sensibilidade para a detecção dos sintomas do quadro ${ }^{22}$. No presente trabalho, foram analisados os dois pontos de corte: $<35^{22} \mathrm{e}<40^{21}$.

\section{Escala de Silhuetas}

Para identificar os diversos componentes da percepção da imagem corporal, adotou-se o instrumento psicométrico Escala de Silhuetas, adaptada e validada para a população brasileira ${ }^{19}$. A partir de análises específicas entre teste e reteste positivos e significativos, a referida escala mostrou-se adequada, tendo em vista a correlação entre o IMC real e o percebido como atual por indivíduos de 18 a 60 anos $^{23}$.

A aplicação da escala citada consiste em apresentar uma série ascendente de silhuetas e pedir que o participante indique a que melhor represente sua silhueta atualmente e, em seguida, que indique a silhueta que gostaria de ter $^{24}$. Cabe registrar que os cartões com as silhuetas foram reproduzidos exatamente nas medidas e cores indicadas no processo de validação do instrumento, a fim de garantir a dimensão adequada para o reconhecimento da forma corporal ${ }^{19}$. Por fim, classifica-se a satisfação ou a insatisfação com a imagem corporal e, para a insatisfação, considera-se o desejo de perder ou ganhar peso ${ }^{25}$.

\section{Análise de dados}

Os valores foram expressos em frequências absoluta (n) e relativa (\%). A associação para análise de variáveis com duas categorias foi realizada pelo teste exato de Fisher. Para análise de variáveis com três ou mais categorias simultaneamente, foi utilizado o teste qui-quadrado e, na presença de significativa associação, foi aplicado o teste exato de Fisher como pós-teste. As análises foram realizadas pelo software estatístico Bioestat 5.0, com nível de significância de 5\%.

\section{RESULTADOS}

Participaram deste estudo 430 indivíduos, sendo 56,7\% (n = 244) do sexo feminino. A idade média foi de 35,3 ([desvio-padrão $(D P)=10,8]$ anos para os homens e de $36,4(D P=11,4)$ anos para as mulheres, com variação de 18,1 a 59,9 anos.

Para as características sociodemográficas, destacaram-se as seguintes distribuições: entre os homens, idade entre 20 e 30 anos (34,9\%), ensino superior completo ou pós-graduação (60,2\%), estado civil casado/união estável $(51,6 \%)$ e renda mensal familiar > 7 salários mínimos (40,9\%). Entre as mulheres, idade entre 20 e 30 anos (31,6\%), ensino superior completo ou pós-graduação (63,5\%), estado civil casado/união estável (48,8\%) e renda mensal familiar > 7 salários mínimos (34,4\%).

As proporções de indivíduos com pontuação indicativa de risco para ortorexia foram: $91,4 \%(n=393)$ para o ponto de corte $<40$ e 54,4\% ( $n=234)$ para o ponto de corte $<35$ (Figura 1, p < 0,0001). Ao considerar a distribuição por sexo, com o ponto de corte $<40,93 \%$ dos homens e $90,2 \%$ das mulheres foram classificados em risco e com o ponto de corte $<35$, as frequências foram de $50,5 \%$ entre os homens e $57,4 \%$ entre as mulheres.

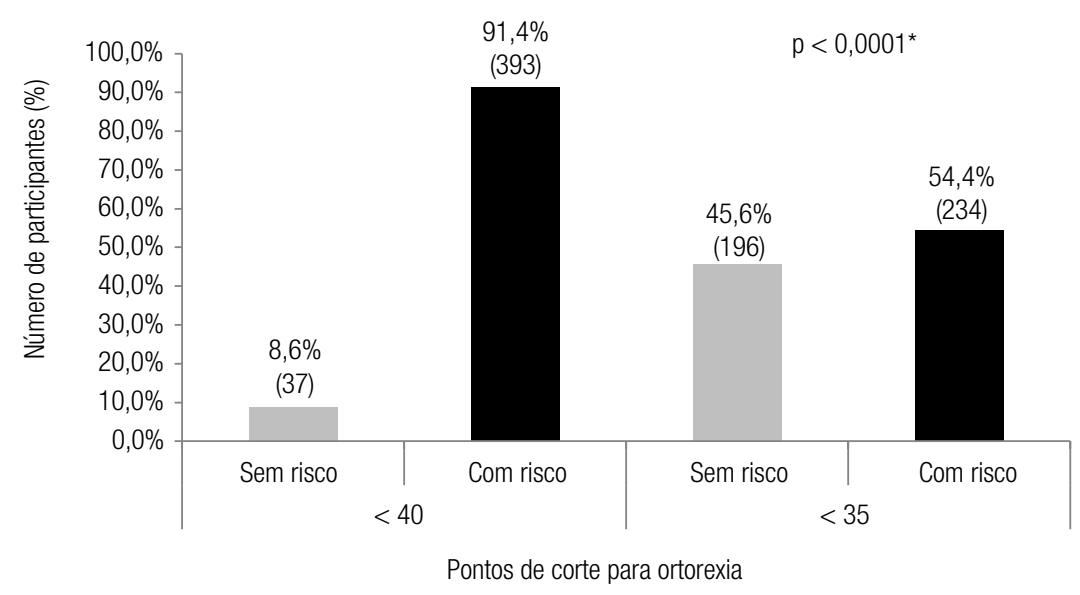

*Associação significativa pelo teste exato de Fisher.

Figura 1. Distribuição dos participantes $(n=430)$ de acordo com o risco para ortorexia pelos pontos de corte $<40$ e $<35-$ Campo Grande (MS), 2018. 
Em relação ao risco de ortorexia, não foi identificada associação entre o sexo e a presença do quadro quando avaliados pelos dois pontos de corte: $<40(p=39)$ e $<35(p=0,17)$.

Os indivíduos pertencentes à faixa etária maior que 40 até 60 anos estão significativamente associados com a presença do risco de ortorexia, ao passo que os indivíduos entre 20 e 40 anos apresentaram uma significativa associação com ausência de risco $(<35)(p=0,0005)$. Quando utilizado o ponto de corte $<40$, nenhuma faixa etária apresentou associação estatística com o risco de ortorexia $(p=0,30)$.
A escolaridade não se mostrou associada ao comportamento ortoréxico, tendo em vista os dois pontos de corte. Do mesmo modo, não houve associação do risco em relação às variáveis estado civil e renda mensal familiar (Tabela 1).

Em relação ao estado nutricional autorreferido (IMC), não houve associação entre as diferentes classificações (baixo peso, eutrofia, sobrepeso ou obesidade) e a presença do risco de ortorexia quando utilizados os dois pontos de corte $(<35, p=0,68, e<40, p=0,69$, respectivamente) (Tabela 2).

Tabela 1. Descrição das frequências relativas (\%) e absolutas (n) dos 430 participantes de acordo com a presença de risco para ortorexia com a utilização dos pontos de corte $<40$ e $<35$ - Campo Grande (MS), 2018

\begin{tabular}{|c|c|c|c|c|c|c|}
\hline \multirow{2}{*}{ Variáveis sociodemográficas } & \multicolumn{2}{|c|}{$\begin{array}{c}\text { Ortorexia }(<40) \\
\%(n)\end{array}$} & \multirow{2}{*}{$\begin{array}{l}\text { Valor } \\
\text { de p }\end{array}$} & \multicolumn{2}{|c|}{$\begin{array}{c}\text { Ortorexia (<35) } \\
\%(n)\end{array}$} & \multirow{2}{*}{$\begin{array}{l}\text { Valor } \\
\text { de } p\end{array}$} \\
\hline & $\begin{array}{c}\text { Sem risco } \\
n=37\end{array}$ & $\begin{array}{c}\text { Com risco } \\
n=393\end{array}$ & & $\begin{array}{c}\text { Sem risco } \\
\mathrm{n}=196\end{array}$ & $\begin{array}{c}\text { Com risco } \\
n=234\end{array}$ & \\
\hline \multicolumn{7}{|l|}{ Sexo } \\
\hline Masculino & $34,1(13)$ & $44,0(173)$ & $0,39^{*}$ & $46,9(92)$ & $40,2(94)$ & $0,17^{\star}$ \\
\hline Feminino & $64,9(24)$ & $56,0(220)$ & & $53,1(104)$ & $59,8(140)$ & \\
\hline \multicolumn{7}{|l|}{ Faixa etária } \\
\hline$\leq 20$ & $8,1(3)$ & $3,8(15)$ & 0,30 & $6,1(12)^{\mathrm{a}}$ & $2,6(6)^{\mathrm{a}}$ & $0,0005^{*}$ \\
\hline$>20 a \leq 40$ & $64,9(24)$ & $60,1(236)$ & & $67,9(133)^{\mathrm{a}}$ & $54,3(127)^{b}$ & \\
\hline$>40 \mathrm{a} \leq 60$ & $27,0(10)$ & $36,1(142)$ & & $26,0(51)^{b}$ & $43,2(101)^{a}$ & \\
\hline \multicolumn{7}{|l|}{ Escolaridade } \\
\hline Analfabeto/Fundamental I Incompleto & $0,0(0)$ & $3,1(12)$ & 0,45 & $2,0(4)$ & $3,4(8)$ & 0,31 \\
\hline Fundamental 2 Completo/Médio Incompleto & $8,0(3)$ & $3,1(12)$ & & $4,0(8)$ & $3,0(7)$ & \\
\hline Médio Completo/Superior Incompleto/Técnico & $30,0(11)$ & $31,8(125)$ & & $31,0(60)$ & $32,5(76)$ & \\
\hline Superior Completo & $35,0(13)$ & $36,4(143)$ & & $41,0(80)$ & $32,5(76)$ & \\
\hline Pós-graduação & $27,0(10)$ & $25,7(101)$ & & $22,0(44)$ & $29,6(67)$ & \\
\hline \multicolumn{7}{|l|}{ Estado civil } \\
\hline Solteiro & $48,6(18)$ & $41,2(162)$ & 0,66 & $48,0(94)$ & $36,8(86)$ & 0,22 \\
\hline Casado/União estável & $43,2(16)$ & $50,6(199)$ & & $45,0(88)$ & $54,3(127)$ & \\
\hline Separado/Divorciado & $8,1(3)$ & $8,1(32)$ & & $7,0(14)$ & $9,0(21)$ & \\
\hline \multicolumn{7}{|l|}{ Renda familiar mensal (em Salários Mínimos) } \\
\hline$\leq 1$ até $\leq 3$ & $24,3(9)$ & $21,4(84)$ & 0,40 & $26,0(51)$ & $17,0(42)$ & 0,12 \\
\hline$>3$ até $\leq 7$ & $48,6(18)$ & $40,5(159)$ & & $40,0(78)$ & $42,3(99)$ & \\
\hline$>7$ & $27,0(10)$ & $38,2(150)$ & & $34,0(67)$ & $39,7(93)$ & \\
\hline
\end{tabular}

Nota: Foi utilizado o teste exato de Fisher para análise de duas categorias da variável e o teste qui-quadrado para análise de 3 ou mais categorias simultaneamente, com pós-teste pelo teste exato de Fisher.

*Associação significativa entre os grupos com e sem risco. As letras nas linhas indicam a classificação do estado nutricional associadas ao risco de ortorexia.

Tabela 2. Distribuição do número de participantes $(n=430)$ de acordo com o índice de massa corporal $(\mathrm{IMC})$, a partir de medidas antropométricas autorreferidas, e o risco para ortorexia segundo os pontos de corte $<40$ e $<35$ - Campo Grande (MS), 2018

\begin{tabular}{|c|c|c|c|c|c|c|}
\hline \multirow{2}{*}{ Estado nutricional autorreferido (IMC) } & \multicolumn{2}{|c|}{$\begin{array}{c}\text { Ortorexia }(<40) \\
\%(n)\end{array}$} & \multirow{2}{*}{$\begin{array}{l}\text { Valor } \\
\text { de } p^{*}\end{array}$} & \multicolumn{2}{|c|}{$\begin{array}{c}\text { Ortorexia (<35) } \\
\%(n)\end{array}$} & \multirow{2}{*}{$\begin{array}{l}\text { Valor } \\
\text { de } p^{*}\end{array}$} \\
\hline & $\begin{array}{c}\text { Sem risco } \\
\mathrm{n}=37\end{array}$ & $\begin{array}{c}\text { Com risco } \\
\mathrm{n}=393\end{array}$ & & $\begin{array}{c}\text { Sem risco } \\
n=196\end{array}$ & $\begin{array}{c}\text { Com risco } \\
\mathrm{n}=234\end{array}$ & \\
\hline Baixo peso & $0,0 \%(0)$ & $2,0 \%(7)$ & \multirow[t]{4}{*}{0,68} & $1,0 \%(2)$ & $2,1 \%(5)$ & \multirow[t]{4}{*}{0,69} \\
\hline Eutrofia & $54,1 \%(20)$ & $45,5 \%(179)$ & & $45,9 \%(90)$ & $46,6 \%(109)$ & \\
\hline Sobrepeso & $35,1 \%(13)$ & $40,2 \%(158)$ & & $39,3 \%(77)$ & $40,2 \%(94)$ & \\
\hline Obesidade & $10,8 \%(4)$ & $12,5 \%(49)$ & & $13,8 \%(27)$ & $11,1 \%(26)$ & \\
\hline
\end{tabular}

* Teste qui-quadrado. 
Quando analisado isoladamente por sexo, o estado nutricional autorreferido (IMC) também não apontou associação entre as diferentes classificações (baixo peso, eutrofia, sobrepeso ou obesidade) e o risco de ortorexia $(<35$ ou $<40$ ).

Ao considerar apenas os indivíduos classificados como ortoréxicos, foi identificada associação entre a classificação em eutrofia e o sexo feminino, assim como entre o sobrepeso e o sexo masculino $(<40)(p<0,0001)$. Para o ponto de corte $<35$, também considerando apenas os indivíduos em risco, foi identificada associação entre a classificação de sobrepeso e o sexo masculino $(p=0,004)$.

Ao analisar o risco de ortorexia com a imagem corporal, expressa pela satisfação ou pelo desejo de perder ou ganhar peso, nos participantes do sexo masculino e feminino não foi constatada associação em ambos os pontos de corte $(<40$ e <35) (Tabela 3).

\section{DISCUSSÃO}

Neste estudo, as frequências indicativas de risco de ortorexia mostraram-se substanciais para ambos os pontos de corte $(<35,54,4 \%$, e $<40,91,4 \%)$. Tais proporções são superiores às observadas em outras pesquisas, também considerando amostra da população geral| ${ }^{10,21,26}$, ou seja, sem grupos considerados de risco, como, por exemplo, atletas ${ }^{27}$, nutricionis$\operatorname{tas}^{28}$, estudantes de Nutrição ${ }^{29}$ e de Medicina ${ }^{30}$, artistas performáticos ${ }^{31}$ e praticantes de yoga ${ }^{32}$. Cabe registrar que no presente estudo se considerou para a coleta de dados local destinado para atividades de lazer, entretenimento e prática de atividade física, aberto ao público de forma gratuita, a fim de possibilitar participantes com características diversas.

A utilização dos dois pontos de corte fundamentou-se em divergências observadas na literatura. Durante a validação do questionário ORTO-15, os autores testaram a eficácia, a sensibilidade, a especificidade e o valor preditivo negativo e positivo e confirmaram a validade do teste para o ponto de corte $<40$, considerando-o apropriado para distinguir indivíduos com e sem ortorexia ${ }^{21}$. Entretanto, outros autores manifestaram preocupação com essa pontuação, por resultarem em falso-positivos, sugerindo que o ponto de corte < 35 evitaria a classificação errônea de indivíduos sem as características do quadro?.

Outros pesquisadores, ao avaliarem as prevalências de acordo com os dois pontos de corte, também notaram diferenças importantes. Com amostra de 92 universitários australianos, ocorreu redução da prevalência de $66 \%(<40)$ para $21 \%(<35)^{33}$, bem como em uma amostra de 275 estudantes norte-americanos, na qual se observou redução da prevalência de $71,2 \%(<40)$ para 22,1\% (<35). Os autores destacaram maior sensibilidade do instrumento com a pontuação $<35$ para a detecção das características ortoréxicas ${ }^{34}$.

Em trabalho realizado com amostra proveniente da população geral, com 404 italianos, identificou-se a prevalência de $6,9 \%$ de comportamento ortoréxico $(<40)^{5}$, proporção extremamente inferior à constatada na presente pesquisa. Contudo, o estudo citado anteriormente foi realizado em outro contexto histórico em relação aos hábitos alimentares,

Tabela 3. Distribuição do número de participantes $(n=430)$ de acordo com a avaliação da imagem corporal e o risco para ortorexia segundo os pontos de corte $<40$ e $<35$ - Campo Grande (MS), 2018

\begin{tabular}{|c|c|c|c|c|}
\hline \multirow{3}{*}{ Risco de ortorexia } & \multicolumn{3}{|c|}{ Imagem corporal } & \multirow{3}{*}{ Valor de $\mathbf{p}^{\star}$} \\
\hline & \multirow{2}{*}{$\begin{array}{l}\text { Satisfeito } \\
\quad \%(n)\end{array}$} & \multicolumn{2}{|c|}{ Insatisfeito } & \\
\hline & & $\begin{array}{c}\text { Desejo de perder peso } \\
\%(n)\end{array}$ & $\begin{array}{c}\text { Desejo de ganhar peso } \\
\text { \% (n) }\end{array}$ & \\
\hline \multicolumn{5}{|l|}{ Ponto de corte $<40$} \\
\hline \multicolumn{5}{|l|}{ Sexo masculino } \\
\hline Sem risco & $7,5 \%$ (3) & $8,1 \%(10)$ & $0,0 \%(0)$ & 0,37 \\
\hline Com risco & $92,5 \%(37)$ & $91,9 \%(113)$ & $100,0 \%(23)$ & \\
\hline \multicolumn{5}{|l|}{ Sexo feminino } \\
\hline Sem risco & $18,8 \%(6)$ & $8,8 \%(17)$ & $5,3 \%(1)$ & 0,17 \\
\hline Com risco & $81,2 \%(26)$ & $91,2 \%(176)$ & $94,7 \%(18)$ & \\
\hline \multicolumn{5}{|l|}{ Ponto de corte $<35$} \\
\hline \multicolumn{5}{|l|}{ Sexo masculino } \\
\hline Sem risco & $47,5 \%(19)$ & $49,6 \%(61)$ & $52,2 \%(12)$ & 0,93 \\
\hline Com risco & $52,5 \%(21)$ & $50,4 \%(62)$ & $47,8 \%(11)$ & \\
\hline \multicolumn{5}{|l|}{ Sexo feminino } \\
\hline Sem risco & $59,4 \%(19)$ & $38,9 \%(75)$ & $52,6 \%(10)$ & 0,06 \\
\hline Com risco & $40,6 \%(13)$ & $61,1 \%(118)$ & $47,4 \%(9)$ & \\
\hline
\end{tabular}


considerando que se vivencia na atualidade a possibilidade de informações disseminadas por diversas formas de mídia sobre comportamentos de valorização à alimentação, denominada de saudável, o que poderia impactar no risco de desenvolvimento da ortorexia.

Também com italianos e não pertencentes a grupos de risco, com o mesmo questionário e com amostra de 177 participantes, foram encontrados sintomas ortoréxicos em $57,6 \%$ dos participantes $(<40)$ e, ao se realizar o ajuste do ponto de corte, a prevalência foi de 11,9\% $(<35)^{10}$.

Em nossa amostra, foi possível observar também uma redução na frequência de indivíduos em risco de ortorexia de $91,4 \%$ ( $n=393$ ) com o ponto de corte $<40$ para $54,4 \%$ ( $n=$ 234) com o ponto de corte $<35$. Contudo, a proporção ainda se mantém expressiva. Dunn e Bratman' apontam, ao considerar outros transtornos alimentares, que a anorexia nervosa e a bulimia nervosa apresentam prevalência relativamente rara (cerca de $2 \%$ para ambas) e que a ortorexia pode retratar possíveis semelhanças com esses transtornos alimentares, dessa forma sugerem que sua prevalência apresente proporções próximas.

Diversos fatores podem contribuir para as diferenças entre os resultados. Para Barrada e Roncero ${ }^{35}$, o ORTO-15 apresenta inconsistências em relação às características psicométricas, como na estrutura interna, nas dúvidas sobre a interpretação das pontuações do questionário e nas limitações da validade do conteúdo. Ainda, é sugerido tratar-se de um instrumento inadequado para distinguir entre alimentação saudável e o comer saudável patológico ${ }^{34}$. Contudo, até o momento, para amostras brasileiras, trata-se do único instrumento disponível para identificar o risco de comportamento ortoréxico.

Outro fator para a divergência de resultados é a falta de critérios diagnósticos bem definidos. Segundo Varga et al. ${ }^{7}$, a ortorexia não é reconhecida como um transtorno alimentar formal. Porém, de acordo com o sistema de classificação da quinta edição do Manual Diagnóstico Estatístico de Transtornos Mentais, a motivação para seguir uma dieta obsessivamente rígida, como algo indispensável, e projetada para promover a boa saúde provavelmente pode ser classificada como transtorno alimentar restritivo/evitativo ${ }^{36}$. Entretanto, como a preocupação com as consequências aversivas da alimentação é tipicamente interpretada como uma reação a um evento traumático prévio, de asfixia ou experiência aversiva como vômitos subsequentes, e não devido à alimentação saudável, é possível que a ortorexia não esteja adequadamente descrita?'

Quanto à influência do sexo, não foi constatada associação entre sexo e comportamento de risco para ortorexia. Esse resultado é similar ao encontrado em uma amostra de alemães, não classificados em grupos de risco para o qua$\mathrm{dro}^{26}$, e corrobora com o resultado encontrado entre estudantes de Ciências do Exercício e de Negócios ${ }^{37}$.
Contudo, em italianos, foi identificada maior frequência entre homens $(11,3 \%)$ do que em mulheres $(3,9 \%)^{5}$, assim como em uma amostra de 878 estudantes de Medicina tur$\cos ^{30}$ e de 459 estudantes universitários norte-americanos ${ }^{38}$. É possível que, com o interesse dos homens quanto à valorização do corpo, da estética e da saúde, se destaquem motivos para seguir uma dieta saudável. Mas, diferentemente das mulheres, por serem novatos nessa situação, tornam-se mais ansiosos e suscetíveis às mensagens de alimentos, de diversas fontes ${ }^{5}$, e acabam apresentando maior risco de desenvolver ortorexia ${ }^{39}$.

Por outro lado, entre 2.130 estudantes universitários italianos, a frequência foi maior entre o sexo feminino $(37,8 \%)$ do que no sexo masculino $(30,7 \%)(<35)^{22}$. Esses achados podem sugerir diferença pouco significativa entre os sexos no quadro de ortorexia, fato que se mostra diferente nos transtornos alimentares, tendo em vista a prevalência de anorexia nervosa com proporção de nove mulheres para cada homem ${ }^{38}$.

Quanto à idade, neste estudo houve associação significativa com a faixa etária de 40 a 60 anos. Outros estudos também encontraram a mesma associação, mas as faixas etárias variaram de acordo com os trabalhos. Estudantes menores de 21 anos estão associados ao quadro quando comparados aos indivíduos de idade superior ${ }^{30}$, assim como mulheres e homens com média de idade de, respectivamente, 33,2 \pm 2,14 e de $36 \pm 17$ anos $^{21}$. Asil e Sürücüoglü40 constataram associação com a faixa etária de $34 \pm$ 11,2 anos e ainda apontaram que a tendência à ortorexia pode aumentar com a idade. Já, em contrapartida, um estudo com participantes com idade média de 50,6 anos não encontrou diferença entre a faixa etária e a ortorexia ${ }^{26}$.

Portanto, sugerimos que a discordância entre a associação das idades e o risco de ortorexia pode ser cultural para as diferentes nacionalidades e ainda regionalizada no próprio país. Além disso, é possível considerar que os mais jovens, especialmente os adolescentes, não demonstram preocupação com a alimentação saudável, o que pode ocorrer entre brasileiros. De acordo com Assis et al. ${ }^{41}$, entre os mais jovens a comida está associada com diferentes contextos e emoções, e os vegetais e alimentos tidos como saudáveis são vinculados às refeições com os pais e ao maior autocontrole, ao passo que a comida não saudável é associada à independência e à integração com outros adolescentes.

No que diz respeito à escolaridade, em nosso estudo não foi detectada associação com as características da ortorexia. Outros estudos apontam que indivíduos com o quadro apresentaram menor número de anos de escolaridade ${ }^{5,26}$. Ramacciotti et $a . .^{10}$ constataram prevalência sensivelmente menor em participantes com graduação e pós-graduação (em ambos os pontos de corte do ORTO-15).

Também não houve associação entre estado civil e ortorexia, corroborando outro estudo que observou não existir 
diferença ${ }^{5}$. Nos trabalhos com amostra classificada nos grupos de risco para ortorexia, os achados diferem. Estudo realizado com 62 indivíduos vegetarianos e não vegetarianos turcos não encontrou associação entre o comportamento ortoréxico e o estado civil ${ }^{42}$. Já entre 1.240 profissionais de saúde italianos e envolvidos em educação nutricional a pontuação total do ORTO-15 foi significativamente menor (indicativo de risco) em indivíduos divorciados ${ }^{43}$.

Não foi encontrada associação entre o risco de ortorexia e a renda familiar no presente estudo, achado também verificado por outro trabalho no qual se constatou que ter maior ou menor nível socioeconômico não foi fundamental para o desenvolvimento de características da ortorexia ${ }^{30}$. Porém, em contradição, outro estudo demonstrou que o nível socioeconômico mais baixo está associado à ortorexia 5 .

Quanto ao estado nutricional, não foi identificada associação com o risco de ortorexia. Contudo, houve associação ao considerar o sexo feminino e eutrofia $(<40)$ e o sexo masculino e sobrepeso $(<40$ e $<35)$.

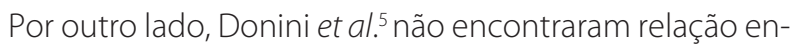
tre os indivíduos em risco para comportamento ortoréxico e IMC. Outros estudos identificaram que, conforme o valor de IMC aumenta, o risco de desenvolver ortorexia também aumenta ${ }^{30,38,40}$. Ressalta-se, segundo a literatura, que a ortorexia se desenvolva a partir de uma tentativa de emagrecimento de indivíduos com excesso de peso, de modo que esses indivíduos tenham uma imagem corporal precisa e não distorcida, voltando-se para a alimentação saudável como forma de alcançar e manter um peso ideal para a saúde física adequada $^{38}$.

Dell'Osso et al. ${ }^{22}$ identificaram que, nos indivíduos em risco para ortorexia, $42,8 \%$ estavam com o IMC indicativo de baixo peso, enquanto $34,3 \%$ estavam com IMC indicativo de excesso de peso. Os autores ainda complementam que esses achados podem sugerir um tipo de continuidade de ortorexia para anorexia nervosa, induzindo a um peso mais leve enraizado na alimentação saudável e impulsionado por um ideal de magreza, resultando em um baixo peso mais grave.

Outra vertente importante relaciona-se à imagem corporal. Neste trabalho não foi observada associação entre o comportamento ortoréxico e a imagem corporal, em ambos os sexos. Entretanto, na literatura constatou-se uma associação positiva entre a preocupação com alimentação saudável e a satisfação com a imagem corporal de mulheres ${ }^{39}$. Sabe-se que as características da ortorexia estão correlacionadas com a distorção da imagem corporal ${ }^{44}$ ou com a baixa satisfação corporal33 $^{33}$, sendo esses indivíduos capazes de se avaliarem maiores ou menores do que realmente são ${ }^{45}$. Ainda, há a possibilidade de que a ortorexia divida similaridades com a anorexia e a bulimia nervosa no que se refere às atitudes em relação à imagem corporal e ao medo de estar acima do peso.

Entretanto, em discordância, é possível que não ocorra distúrbio de imagem corporal associado à ortorexia, pois esse quadro não se relaciona com a perda de peso, mas com ser saudável. Por esse motivo, foi proposto que a ausência do distúrbio de imagem corporal seja um dos critérios para o diagnóstico de ortorexia ${ }^{46}$.

Tendo em vista tratar-se de um estudo com delineamento transversal, norteado para identificar a frequência de risco de ortorexia, cabe destacar também a importância do levantamento de características de natureza sociodemográfica relacionadas ao referido quadro. Assim, além da investigação da frequência, sugerimos o estudo dos fatores associados ao desenvolvimento de atitudes ortoréxicas para melhor compreensão do quadro em diferentes culturas e influências ambientais. Para o presente estudo, as proporções de indivíduos em risco de ortorexia, independentemente do ponto de corte adotado, representam valores expressivos e, assim, requerem cautela, especialmente no que diz respeito à análise dos resultados e repercussões à saúde física e psíquica individual. Tais proporções nos conduzem a instigar pesquisas direcionadas para o aprimoramento de instrumentos para o diagnóstico da ortorexia, que, por sua vez, poderá ser fortalecido pelo futuro reconhecimento do quadro. Quanto ao instrumento adotado, a literatura retrata que a pontuação dos itens do ORTO-15 indica fragilidades ${ }^{47}$, entretanto, como já mencionado, trata-se do único instrumento adaptado para a população brasileira. Ainda como limitações, embora outros estudos também apontem proporções expressivas de indivíduos com comportamentos indicativos de risco de ortorexia, tais resultados podem considerar diferentes instrumentos, o que também indica prudência em análises comparativas. Trata-se de um quadro que requer estudos para categorização exata dos comportamentos envolvidos e respectivo diagnóstico, contudo sabemos que envolve comportamentos cada vez mais frequentes entre indivíduos de todas as idades, o que, mais uma vez, incita estudos específicos. De qualquer forma, este estudo promove avanços ao abordar a ortorexia em amostra não pertencente aos grupos de risco.

\section{CONCLUSÃO}

Os resultados do presente estudo indicam alta frequência de comportamento de risco para ortorexia, porém sem associação com o sexo, escolaridade, estado civil e renda familiar mensal para os pontos de corte adotados. A idade entre $40 \mathrm{e}$ 60 anos associou-se com o risco de ortorexia. Nos indivíduos classificados em risco, foi identificada associação entre eutrofia e sexo feminino $(<40)$ e sobrepeso e sexo masculino $(<40$ e < 35). Sobre a imagem corporal e o risco de ortorexia, não foi encontrada associação. Contudo, diante das frequências constatadas, independentemente dos pontos de corte adotados, ressalta-se a importância de estudos direcionados para o desenvolvimento/refinamento de instrumentos 
e para a identificação do risco de ortorexia, especialmente para a população brasileira, nos diversos estágios de vida e em segmentos não pertencentes aos grupos de risco, com o intuito de conscientização e prevenção de possíveis malefícios à saúde.

\section{CONTRIBUIÇÕES INDIVIDUAIS}

\section{Luís Felipe Lopes Lorenzon e Patrícia Beatriz Pedroso}

Minossi - Participaram da concepção e desenho do estudo, revisão de literatura, coleta de dados, organização, análise e interpretação dos dados, escrita do artigo e aprovação da versão final do artigo.

Giovana Eliza Pegolo - Participou da concepção e desenho do estudo, revisão de literatura, instruções para a coleta de dados, organização, análise e interpretação dos dados, escrita do artigo e aprovação da versão final do artigo.

\section{CONFLITO DE INTERESSES}

Os autores declaram não existir conflito de interesses.

\section{AGRADECIMENTOS}

O presente trabalho foi realizado com o apoio da Fundação Universidade Federal de Mato Grosso do Sul (UFMS)/MEC Brasil.

Agradecemos a todos os participantes da pesquisa e aos colegas do curso de Nutrição, da Faculdade de Ciências Farmacêuticas, Alimentos e Nutrição (FACFAN), da Universidade Federal de Mato Grosso do Sul (UFMS), que auxiliaram na coleta de dados.

\section{REFERÊNCIAS}

1. Dunn TM, Bratman S. On orthorexia nervosa: a review of the literature and proposed diagnostic criteria. Eat Behav. 2016;21:11-17.

2. Bratman S, Knight D. Health food junkies: orthorexia nervosa: overcoming the obsession with healthful eating. New York: Broadway; 2000.

3. Bratman S. What is orthorexia. Orthorexia? 2014. Disponível em: http://www.orthorexia. com/what-is-orthorexia/. Acesso em: 27 nov. 2019.

4. Bratman S. The health food eating disord. [1997?]. Disponível em: https://www. orthorexia.com/original-orthorexia-essay/. Acesso em: 27 nov. 2019.

5. Donini LM, Marsili D, Graziani MP, Imbriale M, Cannella C. Orthorexia nervosa: a preliminary study with a proposal for a diagnosis and an attempt to measure the dimension of the phenomenon. Eat Weight Disord. 2004;9(2):151-7.

6. Cuzzolaro M, Donini LM. Orthorexia by proxy? Eat Weight Disord. 2016;21(4):549-51.

7. Varga M, Thege BK, Dukay-Szabó S, Túry F, Van Furth EF. When eating healthy is not healthy: orthorexia nervosa and its measurement with the ORTO-15 in Hungary. BMC Psychiatry. 2014;14:1-11.
8. Barnes MA, Caltabiano ML. The interrelationship between orthorexia nervosa, perfectionism, body image and attachment style. Eat Weight Disord. 2016;22(1):177-84.

9. Souza AC, Alvarenga MS. Insatisfação com a imagem corporal em estudantes universitários: uma revisão integrativa. J Bras Psiquiatr. 2016;65(3):286-99.

10. Ramacciotti CE, Perrone P, Coli E, Burgalassi A, Conversano C, Massimetti G, et al. Orthorexia nervosa in the general population: a preliminary screening using a self-administered questionnaire (ORT0-15). Eat Weight Disord. 2011;16(2):127-30.

11. Haddad C, Obeid S, Akel M, Honein K, Akiki M, Azar J, et al. Correlates of orthorexia nervosa among a representative sample of the Lebanese population. Eat Weight Disord. 2019;24(3):481-93

12. Souza QJOV, Rodrigues AM. Comportamento de risco para ortorexia nervosa em estudantes de nutrição. J Bras Psiquiatr. 2014;63(3):200-4.

13. Penaforte FRO, Barroso, SM, Araújo ME, Japur CC. Ortorexia nervosa em estudantes de nutrição: associações com 0 estado nutricional, satisfação corporal e período cursado. J Bras Psiquiatr. 2018;67(1):18-24.

14. Plichta M, Jezewska-Zychowicz M. Orthorexic tendency and eating disorders symptoms in Polish students: examining differences in eating behaviors. Nutrients. 2020;12(1):1-17.

15. Costa CB, Hardan-Khalil K, Gibbs K. Orthorexia nervosa: a review of the literature. Issues Ment Health Nurs. 2017;38(12):980-8.

16. Cena H, Barthel F, Cuzzolaro M, Bratman S, Brytek-Matera A, Dunn T, et al. Definition and diagnostic criteria for orthorexia nervosa: a narrative review of the literature. Eat Weight Disord. 2019;24(2):209-46.

17. Brasil. Ministério da Saúde. Conselho Nacional de Saúde. Resolução n 466, de 12 de dezembro de 2012. Aprova diretrizes e normas regulamentadoras de pesquisas envolvendo seres humanos. Brasília, Diário Oficial da União, 12 dez. 2012.

18. Pontes JB, Montagner MI, Montagner MA. Ortorexia nervosa: adaptação cultural ORT0-15. Demetra. 2014;9(2):533-48.

19. Kakeshita IS. Adaptação e validação de Escalas de Silhuetas para crianças e adultos brasileiros [tese]. Ribeirão Preto, SP: Universidade de São Paulo (USP); 2008.

20. Brasil. Orientações para a coleta e análise de dados antropométricos em serviços de saúde: norma técnica do Sistema de Vigilância Alimentar e Nutricional (Sisvan). Brasília, DF: Ministério da Saúde; 2011.

21. Donini LM, Marsili D, Graziani MP, Imbriale M, Cannella C. Orthorexia nervosa: validation of a diagnosis questionare. Eat Weight Disord. 2005;10(2):28-32.

22. Dell'Osso L, Carpita B, Muti D, Cremone IM, Massimetti G, Diadema E, et al. Prevalence and characteristics of orthorexia nervosa in a sample of university students in Italy. Eat Weight Disord. 2018;23(1):55-65.

23. Kakeshita IS, Silda AIP, Zanatta DP, Almeida SS. Construção e fidedignidade teste-reteste de escala de figuras brasileiras para adultos e crianças. Psicol Teor Pesq. 2009;25(2):263-70.

24. Kakeshita IS, Almeida SS. Relação entre índice de massa corporal e a percepção da autoimagem em universitários. Rev Saúde Pública. 2006;40(3);497-504.

25. Nicida DP, Machado KS. 0 uso de duas escalas de silhueta na avaliação da satisfação corporal de adolescentes: revisão de literatura. InterfacEHS. 2014;9(2):21-36.

26. Luck-Sikorski C, Jung F, Schlosser K, Riedel-Heller S. Is orthorexic behavior common in the general public? A large representative study in Germany. Eat Weight Disord. 2018;24(2):267-73.

27. Segura-García C, Papaianni MC, Caglioti F, Procopio L, Nisticò CG, Bombardiere L, et al. Orthorexia nervosa: a frequent eating disordered behavior in athletes. Eat Weight Disord. 2012;17(4):226-33

28. Alvarenga MS, Martins MCT, Sato KSCJ, Vargas SVA, Philippi ST, Scagliusi FB. Orthorexia nervosa behavior in a sample of Brazilian dietitians assessed by the Portuguese version of ORT0-15. Eat Weight Disord. 2012;17(1):29-35.

29. Bo S, Zoccali R, Ponzo V, Soldati L, De Carli L, Benso A, et al. University courses, eating problems and muscle dysmorphia: are there any associations? J Transl Med. 2014;12(1):1-8.

30. Fidan T, Ertekin V, Isikay S, Kirpinar I. Prevalente of orthorexia among medical students in Erzurum, Turkey. Compr Psychiatry. 2010;51(1):49-54.

31. Aksoydan E, Camci N. Prevalence of orthorexia nervosa among Turkish performance artists. Eat Weight Disord. 2009;14(1):33-7.

32. Valera JH, Ruiz PA, Valdespino BR, Visioli F. Prevalence of orthorexia nervosa among ashtanga yoga practitioners: a pilot study. Eat Weight Disord. 2014;19(4):469-72. 
33. Reynolds R. Is the prevalence of orthorexia nervosa in na Australian university population 6,5\%? Eat Weight Disord. 2018;23(4):453-8.

34. Dunn TM, Gibbs J, Whitney N, Starosta A. Prevalence of orthorexia nervosa is less than 1\%: data from a US sample. Eat Weight Disord. 2017;22(1):185-92.

35. Barrada JR, Roncero M. Bidimensional structure of the orthorexia: development and initial validation of a new instrument. An Psicol. 2018;34(2):283-91.

36. American Psychiatric Association. Diagnostic and Statistical Manual of Mental Disorders. 5a ed. Arlington, VA: American Psychiatric Publishing; 2014.

37. Malmborg J, Bremander A, Olsson MC, Bergman S. Health status, physical activity, and orthorexia nervosa: a comparison between exercise science students and business students. Appetite. 2017;109:137-43.

38. Oberle CD, Samaghabadi RO, Hughes EM. Orthorexia nervosa: assesment and correlates with gender, BMI and personality. Appetite. 2017;108:303-10.

39. Brytek-Matera A, Donini LM, Krupa M, Poggiogalle E, Hay P. Orthorexia nervosa and selfattitudinal aspects of body image in female and male university students. J Eat Disord. 2015;3(2):1-8

40. Asil E, Sürücüoglü MS. Orthorexia nervosa in Turkish dietitians. Ecol Food Nutr. 2015;54(4):303-13
41. Assis MM, Penna LF, Neves CM, Mendes APCC, Oliveira RMS, Netto MP. Avaliação do conhecimento nutricional e comportamento alimentar após educação alimentar e nutricional em adolescentes de Juiz de Fora - MG. HU Revista. 2014;40(3):135-43.

42. Çiçekoglu P, Tunçay GY. A comparison of eating attitudes between vegans/vegetarians and non vegans/non vegetarians in terms of orthorexia nervosa. Arch Psychiatr Nurs. 2018;32(2):200-5.

43. Maghetti A, Cicero AFG, D'Ignazio E, Vincenzi M, Paolini B, Lucchin L. Orthorexia prevalence among health care professionals involved in nutrition education: the ADI-0 study. Med J Nutrition Metab. 2015;8(2):199-204

44. Olejniczak D, Bugajec D, Panczyk M, Brytek-Matera A, Religioni U, Czerw A, et al. Analysis concerning nutritional behaviors in the context of the risk of orthorexia. Neuropsychiatr Dis Treat. 2017;13:543-50.

45. Oberle CD, Lipschuetz SL. Orthorexia symptoms correlate with perceived muscularity and body fat, not BMI. Eat Weight Disord. 2018;23(3):363-8.

46. Varga M, Dukay-Szabó S, Túry F, Van Furth FE. Evidence and gaps in the literature on orthorexia nervosa. Eat Weight Disord. 2013;18(2):103-11.

47. Meule A, Holzapfel C, Brandl B, Greetfeld M, Hessler-Kaufmann JB, Skurk T, et al. Measuring orthorexia nervosa: a comparison of four self-report questionnaires. Appetite. 2020;146. 